\title{
Mg-enhanced lateral overgrowth of GaN on patterned GaN/sapphire substrate by selective Metal Organic Vapor Phase Epitaxy
}

\author{
B. Beaumont ${ }^{1}$, M. Vaille ${ }^{1}$, G. Nataf ${ }^{1}$, A. Bouillé ${ }^{1}$, J.-C. Guillaume ${ }^{1}$, P. Vénnègues ${ }^{1}$, S. Haffouz ${ }^{1}$ and \\ Pierre Gibart ${ }^{1}$ \\ ${ }^{1}$ Centre de Recherche sur l'Hétéroépitaxie et ses Applications, CRHEA-CNRS,
}

(Received Wednesday, July 22, 1998; accepted Thursday, September 10, 1998)

Selective and lateral overgrowth by Metal Organics Vapour Phase Epitaxy (MOVPE) was carried out until coalescence to produce smooth and optically flat thick GaN layers. A GaN epitaxial layer is first grown using atmospheric pressure Metalorganic Vapour Phase Epitaxy on a $\{0001\} \mathrm{Al}_{2} \mathrm{O}_{3}$. substrate. Then a $30 \AA$ silicon nitride dielectric film is deposited in-situ by reaction of silane and ammonia to form a selective mask. Afterwards, the openings and the figures in the dielectric films are achieved using standard photolithographic technology. Stripes openings in the mask, revealing free GaN surface, are aligned in the $\langle 10 \overline{1} 0\rangle$ direction. Typical stripes spacing and width are $10 \mu \mathrm{m}$ and $5 \mu \mathrm{m}$ respectively. These patterned layers are further on used for epitaxial regrowth of GaN by MOVPE. The growth anisotropy and therefore the coalescence process is achieved by introducing $(\mathrm{MeCp})_{2} \mathrm{Mg}$ in the vapour phase. A two-step process is reported which allows a dramatic reduction of threading dislocations density not only above the masked areas but also above the windows opened in the mask. With this process, very sharp bound exciton luminescence peaks are measured at low temperature in the overgrown GaN.

\section{Introduction}

Selective epitaxy has been widely developed for III-V semiconductors like GaAs, InP or Si [1]. More precisely, selective epitaxy allows an accurate control of the size and the shape of the overgrowth and therefore leads to the fabrication of quantum structures (quantum wire and quantum dots). Selective epitaxy in heteroepitaxial systems like GaAs/Si [2] or InP/Si [3] has also been widely used to overcome the deleterious effect of the large lattice mismatch.

Selective epitaxy of GaN by both MOVPE and HVPE on patterned GaN on sapphire has been previously reported [4] [5] [6] [7] [8] [9] [10] [11] [12] [13] [14] [15]. Selective epitaxy corresponds to spatially controlled growth of an epitaxial layer through openings in a masking material, which is typically a dielectric such as silicon oxide or nitride. Growth anisotropy corresponds to the occurrence of different growth velocities on different crystallographic planes. This has been observed in HVPE since at least two decades ago. For instance in GaAs there are two orders of magnitude difference between the growth rates of $\{111\}_{\mathrm{Ga}}$ and
$\{1 \overline{1} 1\}_{\text {As }}$. These features are understood by analyzing the orientation dependence of the growth rate associated with surface kinetics. Growth anisotropy in MOVPE occurs only when diffusing molecules encounter different surface orientation within their mean free path $\lambda_{\mathrm{s}}$.

A major problem in selective epitaxy is the ability to provide real selectivity, with growth occurring only in the openings and without any deposits formed on the mask. This can be achieved when the supersaturation of the growth nutrients on the dielectric mask is sufficiently low to prevent any deposition whilst the nucleation barrier on the exposed substrate in the openings is low in comparison. Therefore, in VPE (MOVPE or HVPE), the non-occurrence of growth on the mask depends on the reaction parameters: temperature, pressure and mole fraction of active species.

\section{Growth experiments}

The growth of $\mathrm{GaN}$ is performed in a home-made MOVPE vertical reactor operating at atmospheric pressure. The carrier gas is either pure $\mathrm{N}_{2}$ or a mixture of $\mathrm{N}_{2}: \mathrm{H}_{2} \cdot \mathrm{NH}_{3}$ and the different organometallic species 
(TMGa, $\left.(\mathrm{MeCp})_{2} \mathrm{Mg}\right)$ and diluted silane are introduced into the growth chamber by dedicated lines. A rotating susceptor holds one inch diameter sapphire substrates with (0001) orientation. This reactor is equipped with a $\mathrm{HeNe}$ laser reflectometry set-up used to monitor in-situ the growth process, providing a real time insight on the growth rates and the surface smoothness of the growing samples. In the case of $\mathrm{GaN}$ regrowth on patterned $\mathrm{GaN}$ layers, it has been used to assess the completeness of the coalescence.

\subsection{Preparation of the masked GaN tem- plates}

Patterned substrates used in this work are sketched on figure 1. After deposition of the $\mathrm{GaN}$ buffer layer at $600^{\circ} \mathrm{C}$, a $2-3 \mu \mathrm{m}$ thick $\mathrm{GaN}$ layer is grown at $1080^{\circ} \mathrm{C}$ on a one inch substrate. Detailed description of this growth process is given in [16]. The growth rate is about 2.8 $\mu \mathrm{m} / \mathrm{h}$. X-Ray rocking curves are typically $4 \operatorname{arcmin}$ large. The threading dislocations density determined by TEM observation is in the $10^{8}$ defects $/ \mathrm{cm}^{2}$ range.

The dielectric film was silicon nitride deposited on the GaN MOVPE layer. To this end, after completion of the growth of $\mathrm{GaN}$, diluted silane is introduced in the vapor phase together with $\mathrm{NH}_{3}$, allowing the in situ deposition of a thin $(3 \mathrm{~nm})$ layer. The silicon nitride was patterned using standard photolithography technology, revealing the free surface of the underlying $\mathrm{GaN}$. For the realization of $\mathrm{GaN}$ pseudo-substrates, a $10 \mu \mathrm{m}$ period grating with $5 \mu \mathrm{m}$ wide stripes aligned along $[10 \overline{10}]_{\mathrm{GaN}}$ covering the whole area of the $1 "$ substrate was used. Figure 2 shows the HRTEM image cross section of such a patterned substrate on which GaN was overgrown. The scale is given by the inter-reticular distance in $\mathrm{GaN}$ ( $2.5 \AA$ between horizontal planes). The silicon nitride film is amorphous.

The selectivity of the growth is obtained with a high $\mathrm{H}_{2}$ partial pressure $(0.3 \mathrm{~atm})$ in the growth chamber. When pure $\mathrm{N}_{2}$ is used as carrier, a high density of nuclei is observed. Figure 3 shows the aspect of $\mathrm{GaN}$ regrowth on a mask containing stripes scanning all the crystallographic directions by $5^{\circ}$ increment and an array of circular holes. This figure shows clearly that no parasitic nucleation occurs on the silicon nitride mask nearby the openings.

\subsection{GaN regrowth}

$\mathrm{GaN}$ regrowth conditions used in this work are exactly the same as those used for epitaxy on non masked substrates. The growth temperature is $1080^{\circ} \mathrm{C}$. The total flow is $6 \mathrm{sl} / \mathrm{mn}$ with partial pressures of $\mathrm{NH}_{3}$ and TMGa respectively of 0.3 and $10^{-4} \mathrm{~atm}$. The regrowth is performed at atmospheric pressure.
The shape of undoped GaN ribs after a few minutes of growth on patterned substrates is schematically drawn on figure 4 . The GaN stripes present a trapezoidal cross section with a top $\mathrm{C}$ facet and two slants oriented on the average along $\{11-22\}$. These slants are themselves facetted by $\{10 \overline{1} 1\}$ plane segments due to the high instability of the $\{11-22\}$ growth plane. The slants form an angle $\theta$ of $58^{\circ}$ with the basal C plane.

A schematic cross section of the GaN stripes for longer growth time is shown in figure 5. Dashed lines indicate different intermediate stages of the development of these stripes. During the growth, the two edges of the top $\mathrm{C}$ facet are moving along linear trajectories (provided constant growth rates) indicated by arrows. Note that if the angle $\theta$ is determined only by the structural properties of $\mathrm{GaN}$, the angle $\alpha$ depends also on the anisotropy of the growth i.e. the ratio of vertical to lateral growth rates. A kinematic model involving only the delimiting planes mentioned above yields the following expressions for the coordinates $(\mathrm{x}, \mathrm{z})$ of the points A, B and $\mathrm{C}$ defined on figure 5 :

$$
\begin{gathered}
\text { Point } A:\left(1 / 2 W_{O}, 0\right) \\
\text { Point B: }\left(1 / 2 W_{O}+\left(G_{S}-G_{C} \cos (\theta)\right) t / \sin (\theta), G_{C} t\right) \\
\text { Point C: }\left(1 / 2 W_{O}+G_{S} t / \sin (\theta), 0\right)
\end{gathered}
$$

where $\mathrm{W}_{\mathrm{O}}$ is the width of the stripe opening in the mask, $G_{C}$ and $G_{S}$ are the growth rates in directions normal to the $\mathrm{C}$ facet and the slant respectively and the growth time. The growth rates are assumed to be constant and the linear dependence on time holds as a first order approximation. The points $\mathrm{A}^{\prime}, \mathrm{B}^{\prime}$ and $\mathrm{C}^{\prime}$ are obtained by simple symmetry. From Equation (1) and Equation (3), $\alpha$ is derived as:

$$
\operatorname{tg}(\alpha)=\sin (\theta) /\left(\cos (\theta)-G_{S} / G_{C}\right)
$$

A straightforward interpretation of Equation (4) is that if $G_{S} / G_{C}$ is lower than $\cos (\theta)$ then $\alpha$ is less than $90^{\circ}$ and the two trajectories issued from $\mathrm{A}$ and $\mathrm{A}^{\prime}$ are converging. As observed experimentally, this is the case for the regrowth of undoped $\mathrm{GaN}$ with the growth conditions stated above. This anisotropy is unfavorable for planarization during coalescence because the top $\mathrm{C}$ facet vanishes at the crossing of the two trajectories and the surface of the regrowth is constituted by slants only. An example of such behavior is given in figure 6 showing the cross section of a sample obtained with low growth rate but a long growth time. At this scale the silicon nitride mask cannot be seen. The voids at the coales- 
cence boundary generally reported are not observed too. This is due to the small growth rate for this particular sample.

\subsection{Regrowth of GaN with Mg doping}

Particular growth conditions must be used to achieve favorable anisotropy of the growth i.e. stable $\mathrm{C}$ facets and vanishing slants in order to obtain smooth and planar surface after coalescence is completed. Up to now, the growth temperature was the only parameter reported in the literature that can be adjusted to obtain flat coalesced samples [17]. The cross section of the GaN overgrown stripes tend to be delimited with increasing temperature by vertical sides and a stable $\mathrm{C}$ top facet. In a previous work, we reported that the anisotropy of $\mathrm{GaN}$ in standard growth conditions is dramatically modified by magnesium doping [18]. The main effect of $\mathrm{Mg}$ doping is to reduce to the growth rate $G_{C}$ in [1] direction without substantially modifying the growth rate $\mathrm{G}_{\mathrm{S}}$ of the slants. But the cross section of the GaN:Mg stripes is still delimited by facets having the same crystallographic orientation than in the undoped case.

Therefore, keeping standard growth conditions, the anisotropy $\mathrm{G}_{\mathrm{S}} / \mathrm{G}_{\mathrm{C}}$ can be adjusted by introducing a sufficient amount of $\mathrm{Mg}$ precursor in the vapor phase so that the slants become the fast planes and vanish during coalescence. Figure 7 shows the cross section of a sample obtained by lateral overgrowth of Mg-doped GaN on patterned GaN substrates (5 $\mu \mathrm{m}$ opening with $10 \mu \mathrm{m}$ period). By contrast to figure 6 , the surface of the overlayer is smooth and we observe clearly voids buried at the foot of coalescence boundaries. The planarization was completed after about 2 hours growth run. Figure 8 illustrate diagrammatically how the shape of the stripes is evolving in the Mg doped overgrowth case. $G_{S} / G_{C}$ is now greater than $\cos (\theta), \alpha$ is more than $90^{\circ}$ and the geometrical result is that the trajectories of the top facet edges diverge correspondingly. An expansion of the $\mathrm{C}$ facet is obtained.

\subsection{A two-step lateral overgrowth process}

Samples, as shown in figure 7, covering one inch diameter patterned GaN/sapphire substrate meet the flatness requirement for pseudo $\mathrm{GaN}$ substrates to be used further for the growth of devices structures. But the most important feature expected from the lateral overgrowth process is a large reduction of the density of threading dislocations which are mainly responsible for the short life time of high current injection devices or for the low mobility of undoped GaN. The target is to obtain GaN layers free of threading defects within areas compatible with devices geometry. In $\mathrm{GaN}$, it is known that most of the threading defects are due to low angle boundaries existing within the mosaic structure of the epilayers.
Moreover these defects thread in the growth direction along the $\mathrm{C}$ axis and their density is not reduced substantially when increasing the thickness of the sample.

On one hand, we observed [14] by HRTEM on cross sections of GaN samples grown on patterned substrates with anisotropy unfavorable to planarization (i.e. no doping with standard growth conditions) that (i) the defect lines above the masked area lie parallel to the basal plane (0001), (ii) the $90^{\circ}$ line bending is initiated above the windows in the mask. This was also mentioned in the literature [19] where the bending points of the defect lines are reported to be contained in the trace of the $\{10 \overline{1} 1\}$ slants. On the other hand, when the growth anisotropy is favorable for the planarization (high growth temperature or with $\mathrm{Mg}$ doping in our work), the overgrown $\mathrm{GaN}$ is reported to be free of emerging defects above the masked areas but the threading defects originated from the underlying GaN still propagate vertically through the mask opening and then emerge at the top surface of the overgrowth.

Following these experimental facts, we have investigated a two-step process for the lateral overgrowth of $\mathrm{GaN}$. In the first step, undoped $\mathrm{GaN}$ is overgrown until the top $\mathrm{C}$ facets vanish. In the second step, $\mathrm{Mg}$ is introduced to produce the coalescence with planarization. The first step is intended to initiate defects lines bending over the openings of the mask whereas the second step aims to planarization. This two step process is schematically shown on figure 9.

Figure 10 shows the SEM view of the cross section of a sample grown following this two steps process. No metallization was applied in order to resolve the resistivity contrast between undoped and $\mathrm{Mg}$ doped $\mathrm{GaN}$. The growth run has been stopped before completeness of the planarization. During step (1), a surface having a "saw tooth" section is achieved with peaks and valleys. As soon as the $\mathrm{Mg}$ dopant is introduced, as discussed above, the slowly growing $\mathrm{C}$ top facets reappear. Due both to the deep and sharp V-shaped valley created during (1) and to the sudden change in the growth anisotropy in (2), limited diffusion of the nutrients results in the creation of voids in the valleys [1]. This phenomenon can be avoided by stopping step (1) before two neighboring stripes come in contact. Then the valley is rather U-shaped, with wide angles formed between the two slants and the mask as sketched in figure 5. On figure $10, t_{1}$ and $t_{2}$ represent the lateral expansion of the slants without and with $\mathrm{Mg}$ doping respectively. They are found to be proportional to the growth time allowed for each step and this tends to prove that $\mathrm{Mg}$ affects only the growth on $\mathrm{C}$ plane whereas the growth rate of the slant is not notably modified $\left(\mathrm{G}_{\mathrm{S}} \sim 0.7 \mu \mathrm{m} / \mathrm{h}\right.$ for this sample). $t_{3}$ is the expansion of the $\mathrm{C}$ top facet after its 
reappearance, $t_{2} / t_{3}$ gives an estimate of 3.2 for the growth rates ratio $\mathrm{G}_{\mathrm{S}} / \mathrm{G}_{\mathrm{C}}$. The average measurements of $\alpha$ give for $G_{S} / G_{C}$ a value of about 3 in good agreement with the previous one.

Finally figure 11 is the SEM tilted view of a sample on $\mathrm{GaN}$ overgrown with the two step process with complete planarization and terminated by an undoped $\mathrm{GaN}$ layer. Very smooth surfaces are obtained. The arrow points to an hexagonal pit formed at the coalescence boundary. Along the cross section a periodic grating of voids is seen. This sample is about $16 \mu \mathrm{m}$ thick.

\section{Assessment of the two-step GaN overgrowth.}

\subsection{Mg incorporation}

Figure 12 is the SEM cross section of two-step GaN overgrowth on $\mathrm{GaN}$ masked layer observed at low temperature $(100 \mathrm{~K})$ without metallization. The structure of the sample consists in a masked GaN layer grown in a first run on sapphire, the two-step GaN and finally an undoped $\mathrm{GaN}$ are deposited on top in a second run. For some samples, the undoped top layer has been deposited during a third run after cleaning the reactor in order to limit the contamination by $\mathrm{Mg}$ due to a memory effect.

Dashed vertical white lines are the coalescence boundaries separated by a $10 \mu \mathrm{m}$ period. Plain white lines localize the border between steps 1 and 2. At low temperature, the volume filled during step 2 appears to be inhomogeneous. It is constituted by two zones delimited by a zigzag line along which the two dashed arrows are drawn. These volumes are labeled $2^{\mathrm{S}}$ and $2^{\mathrm{T}}$. Volume $2^{\mathrm{S}}$ is filled during lateral overgrowth by expansion of the slants whereas $2^{\mathrm{T}}$ during expansion of the top $\mathrm{C}$ facets. The apparent inhomogeneity is confirmed by cathodoluminescence study of the cross section. The three spectra plotted in the inset are measured in the cross section of volumes $1,2^{\mathrm{T}}$ and $2^{\mathrm{S}}$. Volume 1 has a typical spectrum with bandedge emission of undoped GaN. Spectra of the volumes $2^{\mathrm{S}}$ and $2^{\mathrm{T}}$ correspond to $\mathrm{GaN}$ with low and high concentration of $\mathrm{Mg}$ dominated respectively by donor-acceptor pairs recombination and the deep blue luminescence band [20]. We have checked by cathodoluminescence mapping that $\mathrm{Mg}$ doped $\mathrm{GaN}$ grown during step 2 is divided in two regions only. Above the zigzag line, the white gray zone is uniformly highly $\mathrm{Mg}$ doped $\mathrm{GaN}$ whereas below that line the darker gray zone corresponds to uniformly lightly $\mathrm{Mg}$ doped GaN. Most probably the zigzag line is a visualization of the trajectory of the $\mathrm{C}$ facets edges during the regrowth as the kinematic model predicts.

The origin of the inhomogeneous $\mathrm{Mg}$ distribution may be due to an anisotropy of its incorporation on dif- ferent crystalline growth planes. But the difference in doping level may also be related to the observed difference in growth rates. The informations given by luminescence are only qualitative and SIMS analysis will be performed to check if the $\mathrm{Mg}$ atomic concentration in volume $2^{\mathrm{S}}$ and $2^{\mathrm{T}}$ are inversely proportional to their growth rates.

\subsection{Observation of the threading defects bending}

Figure 13 is the cross section TEM image of a two-step $\mathrm{GaN}$ overgrown on a patterned substrate. Threading dislocations present in the underlying masked GaN propagate vertically in the overgrown $\mathrm{GaN}$ through the mask opening. But all of them bend by $90^{\circ}$ at points indicated by short arrows. During subsequent growth, the bent defect lines merge into the coalescence boundary. It is noticed that these points tend to organize along two lines visualized on the figure by the converging long arrows. A clear planar symmetry exists in this bending phenomenon. This reflects the symmetrical growth on both side of a plane parallel to and equidistant from the coalescence boundaries delimiting the periodic stripe patterns. The lines drawn through the bending points are likely to be the trajectory of the edges of the vanishing top $\mathrm{C}$ facets. This suggests that bending occurs when a threading dislocation line crosses the right or left moving edge of the top $\mathrm{C}$ facet. As a result, when the top $\mathrm{C}$ facet has fully vanished at the end of step 1 of the process, all threading lines have been crossed and bent by $90^{\circ}$ either to left or to right depending on their relative position from the middle of the mask opening. Therefore after that time, during step 2, the whole upper volume of the overgrowth comprised between the coalescence boundaries is free of vertically threading defects as observed by TEM observation on plane views.

Though the exact mechanism responsible for the bending is not known yet, a tentative view of this twostep process could be as schematically drawn on figure 14. At the end of step 1, during which the lateral growth is negligible, the slant is constituted by the cross section of the misoriented columns arising from the $\mathrm{GaN}$ masked layer. During step 2, the growth is mainly lateral and the columnar mosaic [21] is replaced by a lamellar stacking with low angle misorientations being accommodated in the basal plane.

\subsection{Luminescence of GaN by epitaxial lat- eral overgrowth}

Figure 15 shows the luminescence spectrum of undoped $\mathrm{GaN}$ grown on top of a pseudo-substrate obtained by the two-step process described above after reactor cleaning to minimize $\mathrm{Mg}$ contamination. This kind of spectrum is representative and has been observed with small variation for different samples grown following the same pro- 
cess. The main feature is the sharpness of the peaks. $\mathrm{GaN}$ layers grown directly on sapphire in the same reactor have FWHM of about $3 \mathrm{meV}$ in the best cases. FWHMs below $1 \mathrm{meV}$ are achieved for bound excitons. This is comparable to results reported for homoepitaxial $\mathrm{GaN}$ [22] and HVPE GaN [23]. The free excitons A and $\mathrm{B}$ are well resolved as well as the three emission lines labeled $\mathrm{A}^{*}$ attributed to excited states of A following [23].

\section{Conclusion}

We have developed a two-step growth process to obtain pseudo GaN substrates by epitaxial lateral overgrowth on patterned GaN deposited on sapphire. In the first step, the vertical threading defects are bent by $90^{\circ}$ during the shrinking of the top $\mathrm{C}$ facet delimiting the $\mathrm{GaN}$ ribs. In the second step, owing to the effect of Mg doping on the growth anisotropy, planarization of the surface is achieved during coalescence. This two-step process results in a reduced defect density over the masked area but also over the openings in the masked. The area available for growth of devices structures is then comprised between the coalescence boundaries in which the emerging defects are confined. It is shown that the luminescence of $\mathrm{GaN}$ is strongly improved as far as intensity and sharpness of the emission lines are concerned.

\section{ACKNOWLEDGMENTS}

The authors would like to thank M. Passerel, M. Leroux, M. Teisseire and G. Neu for their helpful contribution to this work. Special thanks are also expressed to Y. Genuist from LSP-CNRS for cathodoluminescence experiments. This work is supported by an EU contract ESPRIT LTR-LAQUANI 20968.

\section{REFERENCES}

[1] L. Jastrzebski, J. Cryst. Growth 63, 493 (1983).

[2] D. Pribat, B. Gerard, M. Dupuy, P. Legagneux, Appl. Phys. Lett. 60 , 2144 (1992).

[3] O. Parillaud, E. Gil-Lafon, B. Gérard, P. Etienne, D. Pribat, Appl. Phys. Lett. 68,2654 (1996).

[4] Y. Kato, S. Kitamura, K. Hiramatsu, N. Sawaki, J. Cryst. Growth 144 , 133 (1994).

[5] S. Kitamura, K. Hiramatsu, N. Sawaki, Jpn. J. Appl. Phys. 24, L1184 (1995).

[6] T Detchprohm, T Kuroda, K Hiramatsu, N Sawaki, H Goto, Inst. Phys. Conf. Ser. 142 , 859-862 (1996).

[7] D. Kapolnek, S. Keller, R. Vetury, R.D. Underwood, P. Kozodoy, S.P. DenBaars, U.K. Mishra, Appl. Phys. Lett. 71 , 1204-1206 (1997).

[8] O-H Nam, MD Bremser, TS Zheleva, RF Davis, Appl. Phys. Lett. 71 , 2638-2340 (1997).
[9] Tsvetanka S. Zheleva, Ok-Hyun Nam, Micheal D. Bremser, Robert F. Davis, Appl. Phys. Lett. 71, 2472-2474 (1997).

[10] O Nam, MD Bremser, BL Ward, RJ Nemanich, RF Davis, Jpn. J. Appl. Phys. 36, L532 (1997).

[11] A Usui, H Sunakawa, A Sakai, AA Yamaguchi, Jpn. J. Appl. Phys. 36, L899 (1997).

[12] Hugues Marchand, J.P. Ibbetson, Paul T. Fini , Peter Kozodoy, S. Keller, Steven DenBaars, J. S. Speck, U. K. Mishra, MRS Internet J. Nitride Semicond. Res. 3, 3 (1998).

[13] Zhonghai Yu , M.A.L. Johnson, T. Mcnulty, J.D. Brown, J.W. Cook,Jr, J.F. Schetzina, MRS Internet J. Nitride Semicond. Res. 3, 6 (1998).

[14] B. Beaumont, P. Gibart, M. Vaille, S. Haffouz, G. Nataf, A. Bouillé, J. Cryst. Growth 189/190 , 97 (1998).

[15] S Nakamura, M Senoh, S Nagahama, N Iwasa, T Yamada, T Matsushita, H Kiyoku, Y Sugimoto, T Kozaki, H Umemoto, M Sano, K Chocho, Appl. Phys. Lett. 72, 211213 (1998).

[16] P. Vennegues, B. Beaumont, S. Haffouz, M. Vaille, P. Gibart, J. Cryst. Growth 187 , 167 (1998).

[17] O-H Nam, T. S. Zheleva, MD Bremser, RF Davis, J. Electron. Mater. 27, 233 (1998).

[18] B. Beaumont, S; Haffouz, P. Gibart, Appl. Phys. Lett. 72,921 (1998).

[19] A Sakai, H Sunakawa, A Usui, Appl. Phys. Lett. 71, 2259-2261 (1997).

[20] M. Leroux, B. Beaumont, N. Grandjean, P. Lorenzini, S. Haffouz, P. Vennegues, J. Massies, P. Gibart, Mater. Sci. Eng. B 50, 97 (1997).

[21] F. A. Ponce, MRS Bull. 22 , 51-57 (1997).

[22] H. Teisseyre, G. Nowak, M. Leszczynski, I. Grzegory, M. Bockowski, S. Krukowski, S. Porowski, M. Mayer, A. Pelzmann, Markus Kamp, K. J. Ebeling, G. Karczewski, MRS Internet J. Nitride Semicond. Res. 1, 13 (1996).

[23] A. Hoffmann, L. Eckey, Mater. Sci. Forum 264/268 , 1259 (1998).

\section{FIGURES}

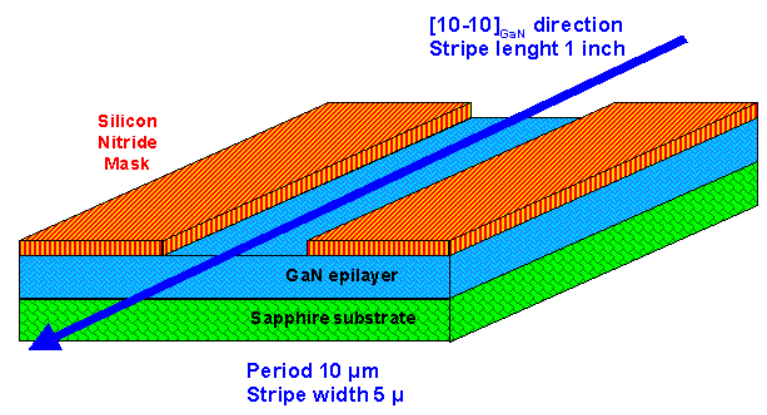

Figure 1. Structure of the patterned $\mathrm{GaN}$ on sapphire template typically used to study epitaxial lateral overgrowth of GaN. A $30 \AA$ amorphous silicon nitride film is formed in situ immediately after growing the GaN epilayer. Windows in the mask are opened by reactive ion etching. 


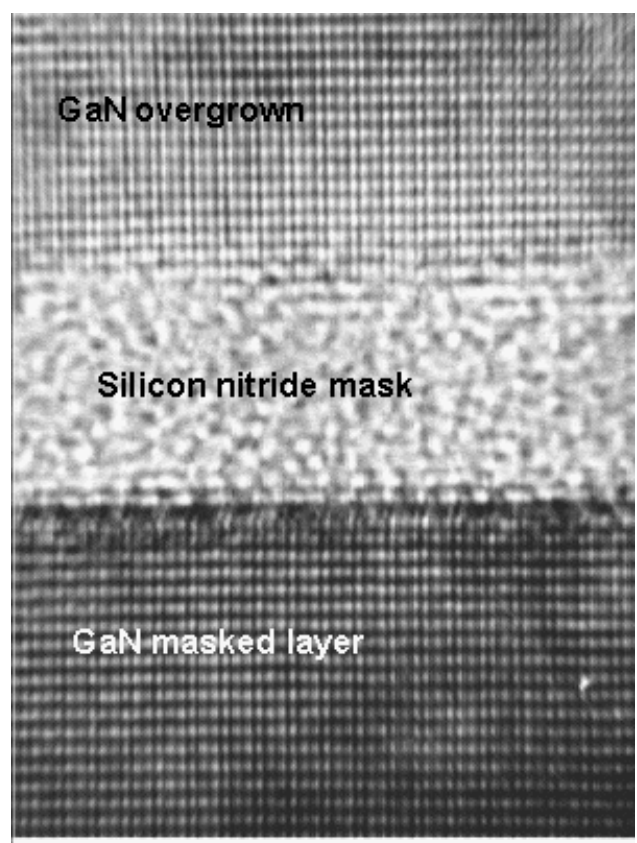

Figure 2. HR-TEM view of a cross section of the GaN overgrown on a masked and patterned GaN layer. The silicon nitride mask is about $30 \AA$ thick.

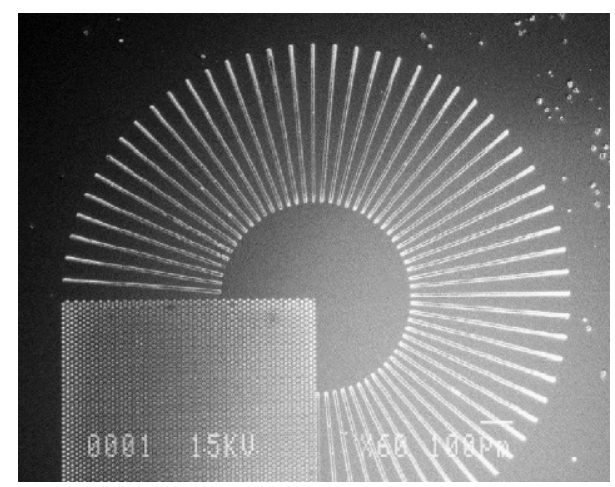

Figure 3. SEM top view of a star pattern mask. No parasitic nuclation is observed in the inner masked area whose diameter is $650 \mu \mathrm{m}$ nor between the stripes. The marker is $100 \mu \mathrm{m}$.

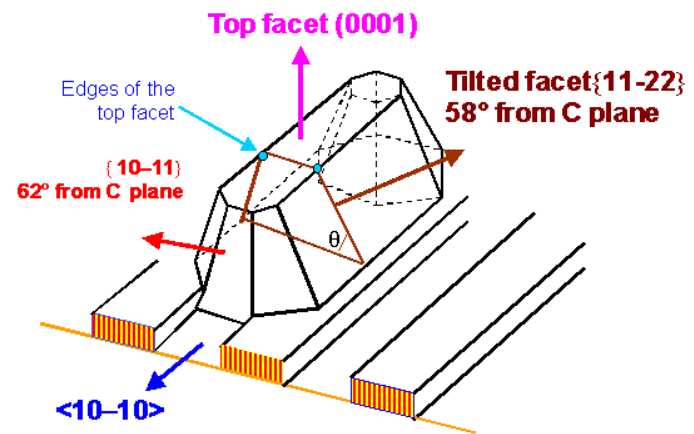

Figure 4. Schematic view of the GaN stripes at the beginning of the regrowth on the patterned substrates of figure 1 .

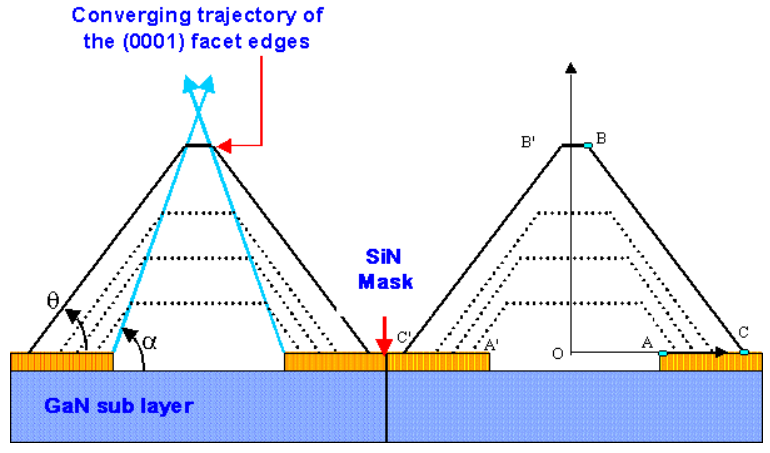

Figure 5. Schematic cross section of the GaN stripes for longer growth time. Dashed lines are different intermediate stages of the development of these stripes. During the growth, the two edges of the top $\mathrm{C}$ facet are moving along linear trajectories (provided constant growth rates) indicated by arrows. For undoped $\mathrm{GaN}$, the top $\mathrm{C}$ facet vanishes above the crossing of the two trajectories. Note that if $\theta$ depends only on the structural properties of $\mathrm{GaN}, \alpha$ is determined by the anisotropy of the growth i.e. the ratio of vertical to lateral growth rates. 


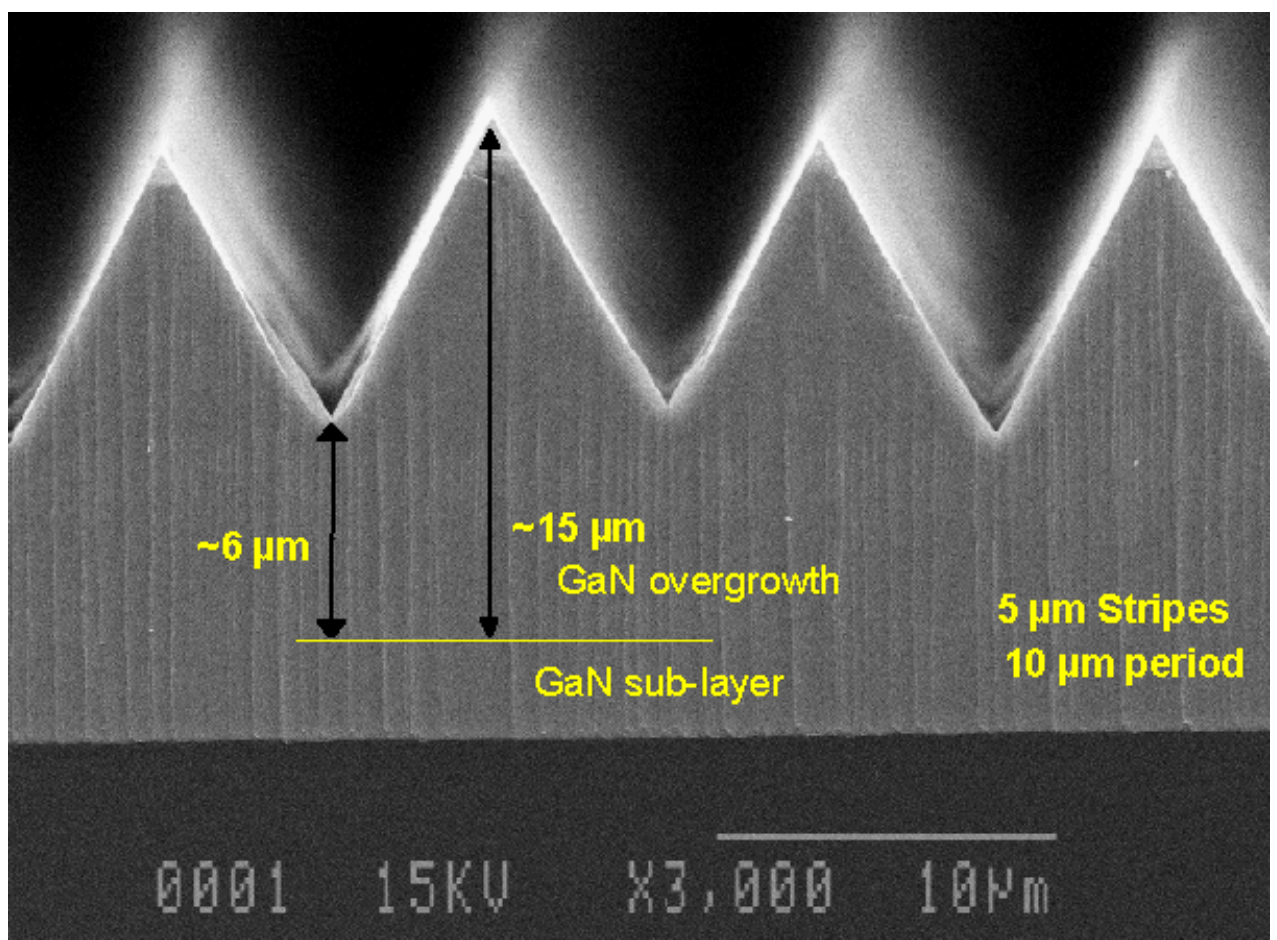

Figure 6. SEM cross section of lateral overgrowth of undoped $\mathrm{GaN}$ in standard conditions. Though perfect coalescence is obtained (without voids at the coalescence boundaries due to very slow growth rates), no smoothing is achieved.

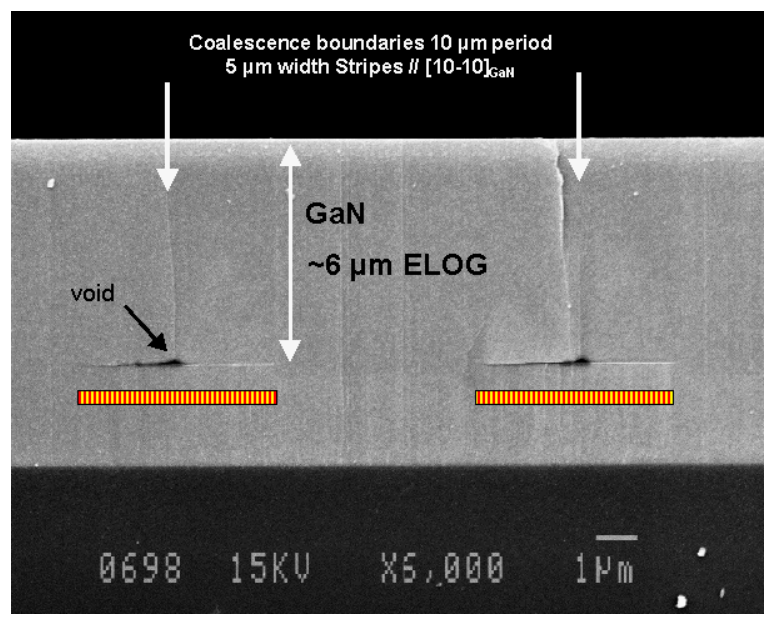

Figure 7. SEM cross section on $\mathrm{Mg}$ doped $\mathrm{GaN}$ overgrown at $1080^{\circ} \mathrm{C}$ on patterned substrate. Flat surface, contrasting with figure 6 , is obtained due to the favorable growth anisotropy induced by the $\mathrm{Mg}$ doping. The mask appears as a very thin line above the dashed filled rectangle.

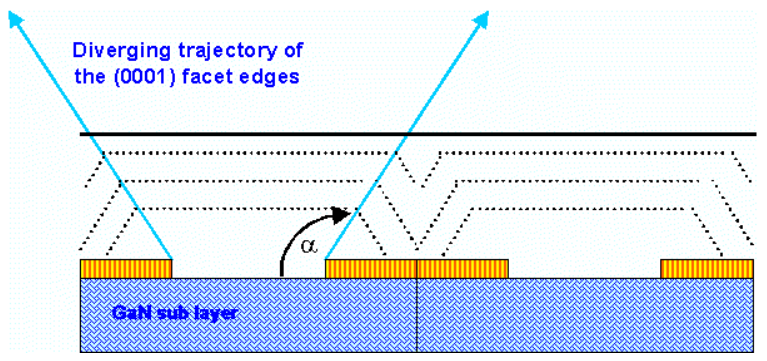

Figure 8. Schematic cross section of the $\mathrm{Mg}$ doped $\mathrm{GaN}$. During the growth, the two edges of the top $\mathrm{C}$ facet still move along linear trajectories but due to doping, these trajectories are now diverging and the two slants are vanishing during coalescence as shown by the intermediate shapes (dashed lines). 


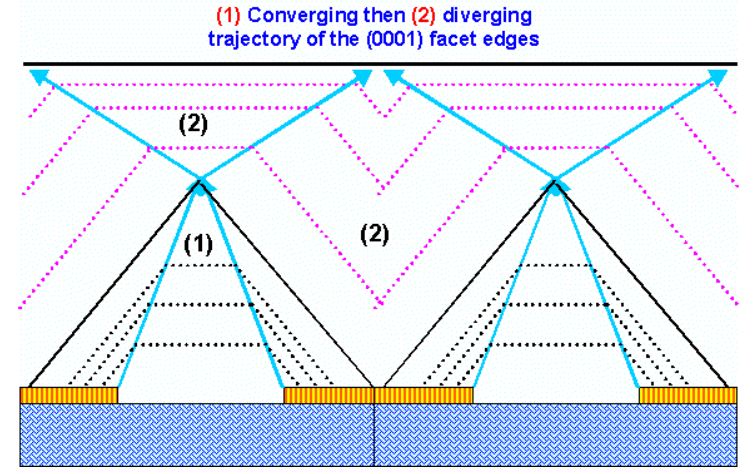

Figure 9. Schematic principle of the two step GaN lateral overgrowth process. In step (1), undoped $\mathrm{GaN}$ is grown at $1080^{\circ} \mathrm{C}$. In these conditions, the top $\mathrm{C}$ facets vanish. In step (2), under the same growth conditions but due to $\mathrm{Mg}$ doping, the $\mathrm{C}$ facets become the slow planes therefore reappearing and leading to planarization by expansion. The arrows represent the trajectories of the $\mathrm{C}$ facet edges. 


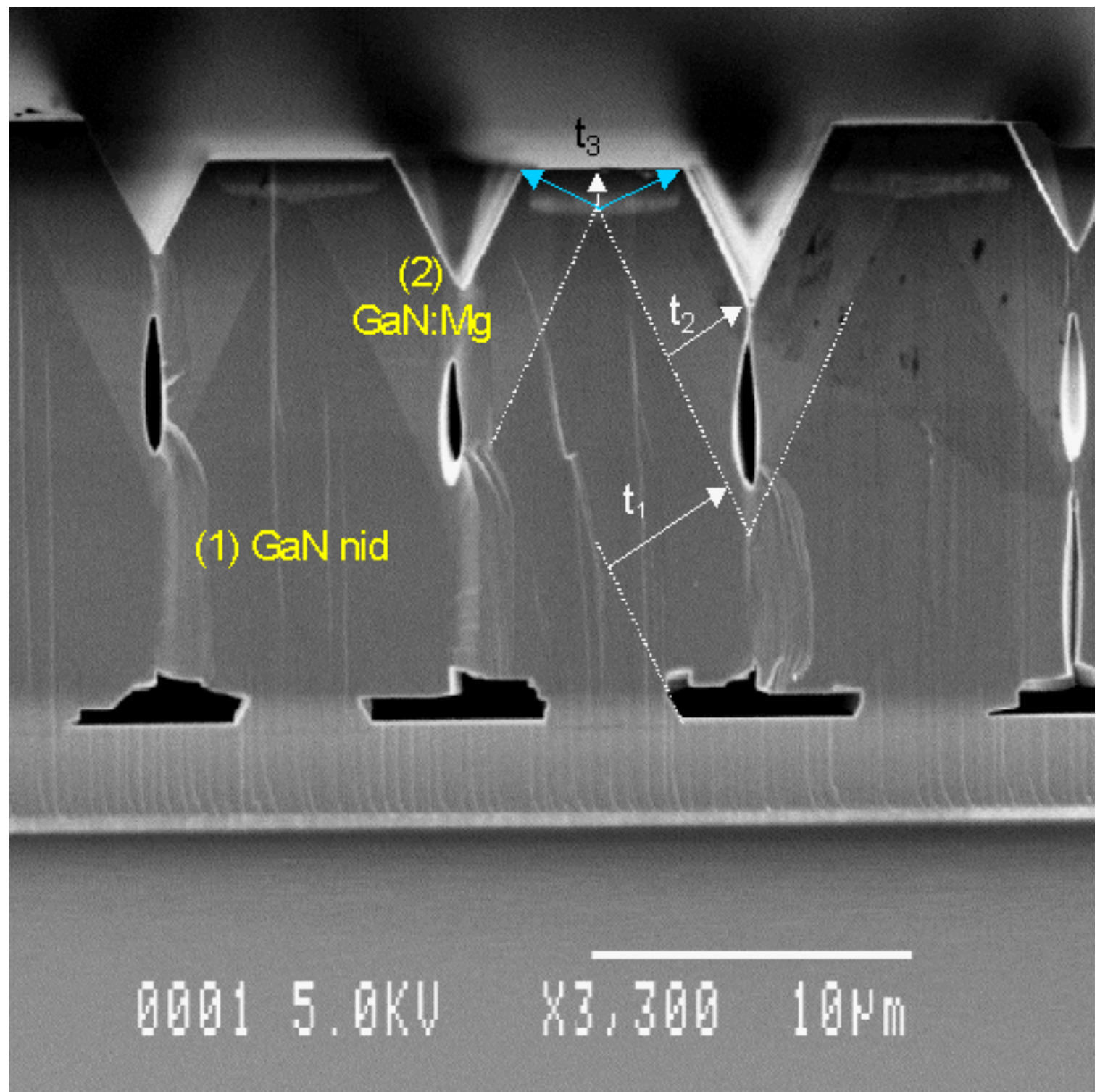

Figure 10. SEM cross section view of lateral overgrown GaN following the two step process. The sample was not metallized in order to observe resistivity contrast between (1) undoped (dark gray) and (2) Mg doped (light gray) GaN. In (1) a peaky surface as in figure 6 is obtained, the dashed line was drawn as a guide for the eye. In (2) the top $C$ facets have reappeared and expand following the arrows. Note that voids are created in the deep valley of (1) during (2) mainly due to diffusion limited growth. 


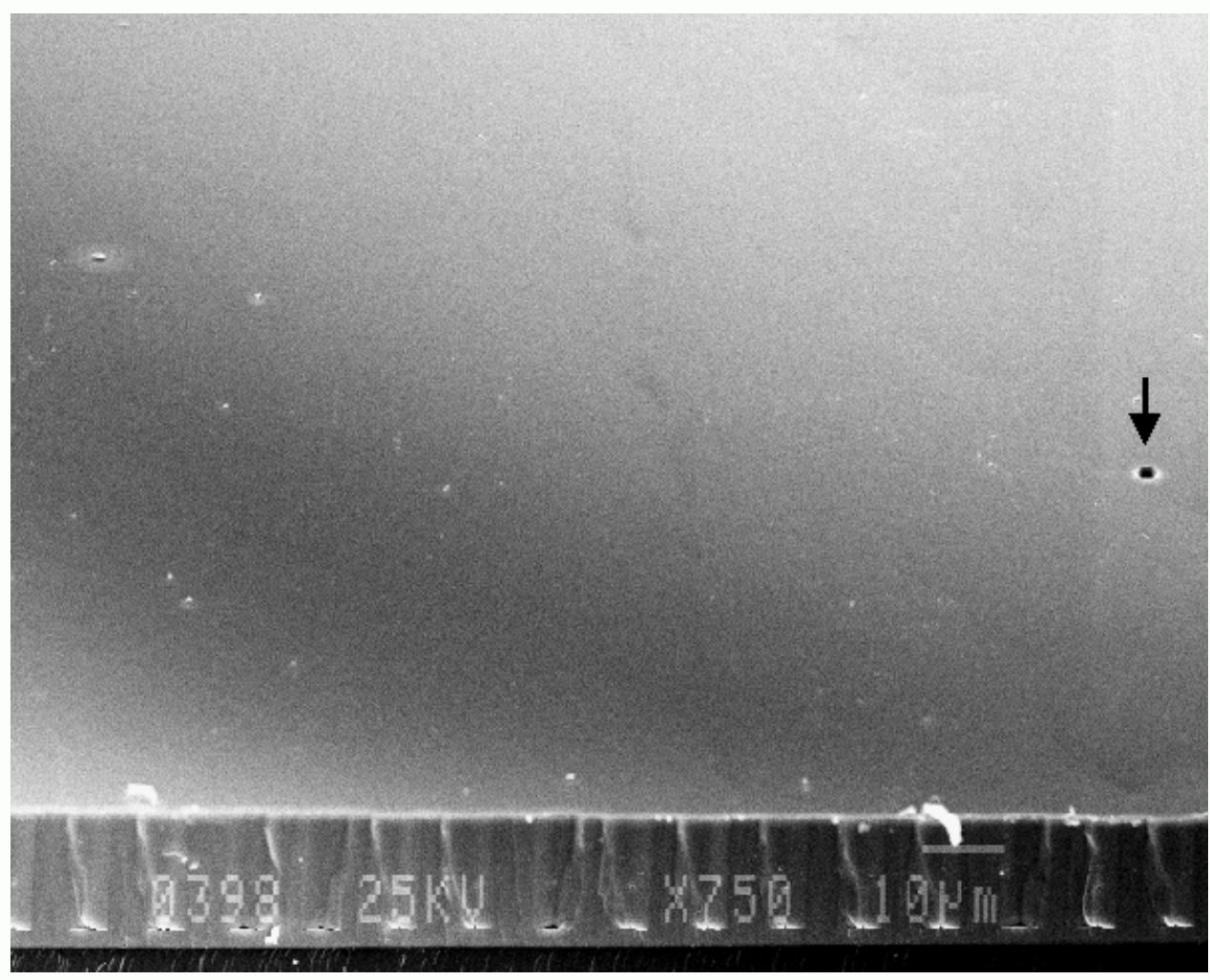

Figure 11. SEM tilted view of the cross section, perpendicular to the stripes direction, of GaN overgrown using the two steps process on patterned GaN/Saphir substrate. Very smooth surfaces are obtained. The arrow points to an hexagonal pit formed at the coalescence boundary. Along the cross section a periodic grating of voids is seen. 

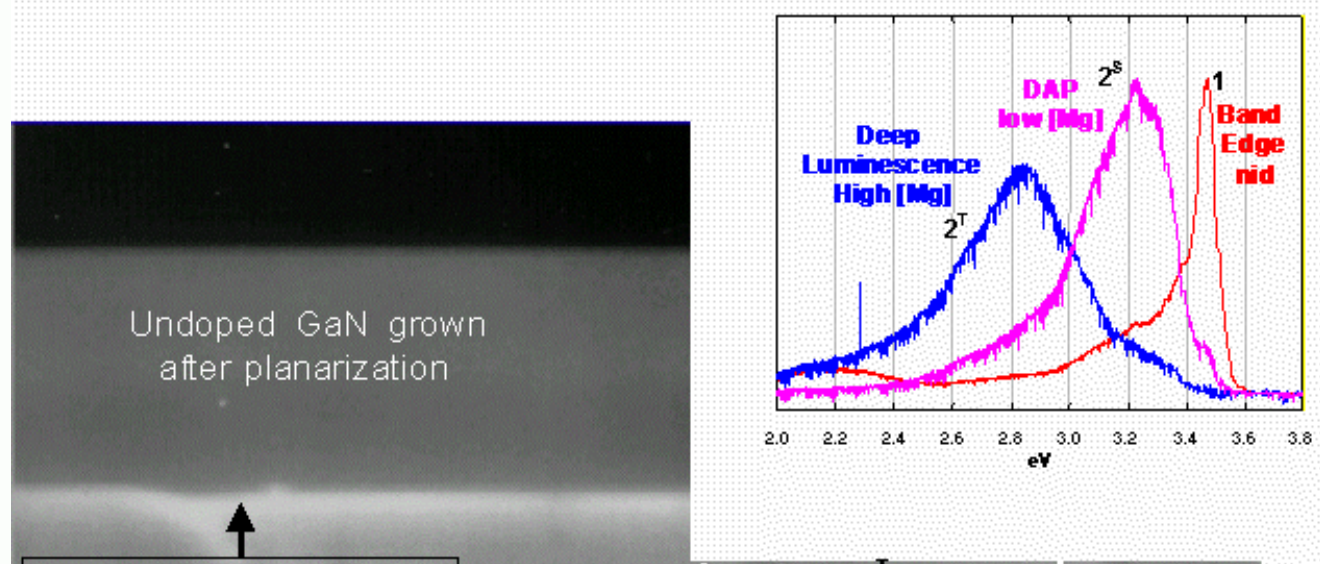

\section{Two-step GaN overgrowth}

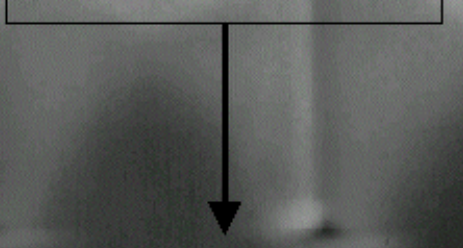

\section{GaN masked layer}

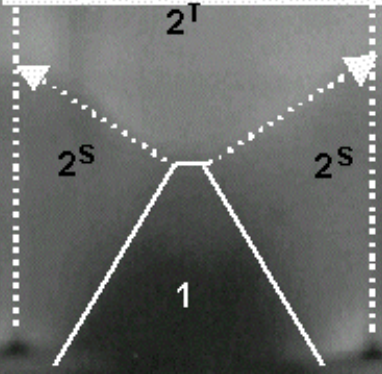

Figure 12. SEM cross section of two-step GaN overgrowth on GaN masked layer observed without metallization at low temperature. Dashed vertical white lines are the coalescence boundaries. Plain white lines localize the border between steps 1 and 2 . The volume filled during step 2 appears to be inhomogeneous, the volume labeled $2^{\mathrm{S}}$ and $2^{\mathrm{T}}$ are filled by expansion of the slants and the top $\mathrm{C}$ facets respectively. This is confirmed by cathodoluminescence spectra plotted in the inset. Volume 1 has a typical spectrum of undoped $\mathrm{GaN}$ whereas spectra of the volumes $2^{\mathrm{S}}$ and $2^{\mathrm{T}}$ correspond to $\mathrm{GaN}$ with low and high concentration of Mg respectively. 


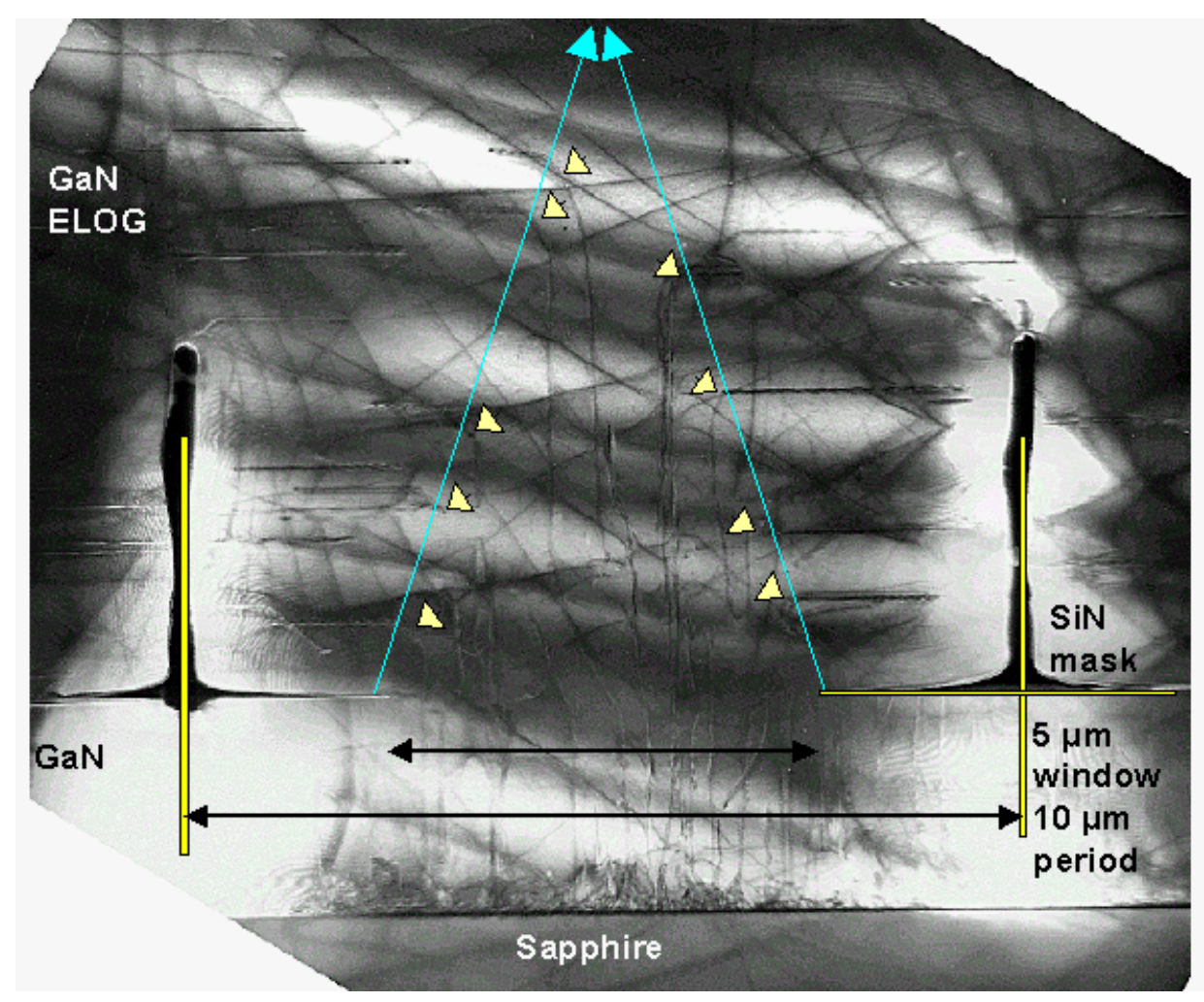

Figure 13. TEM cross section view of GaN overgrown with the two-step process.

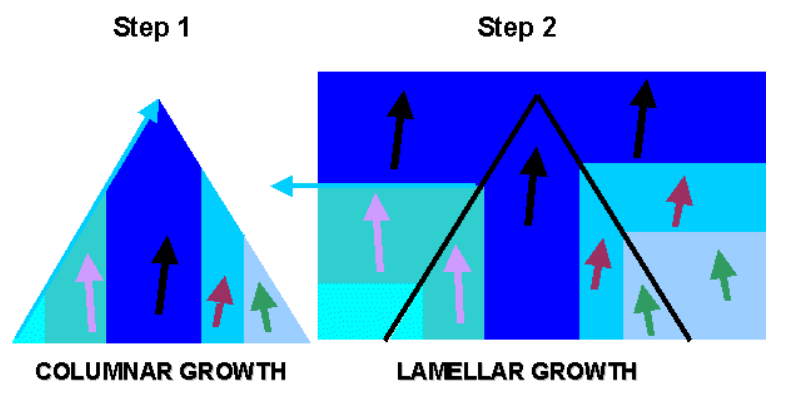

Figure 14. Schematic view of the two-step process. At the end of step 1 with essentially no lateral expansion, the template for the next growth step is formed by section of the misoriented columns by the slants. During step 2 where anisotropy force mainly lateral expansion, the misorientation of the template could be replicated in stacked lamellae.

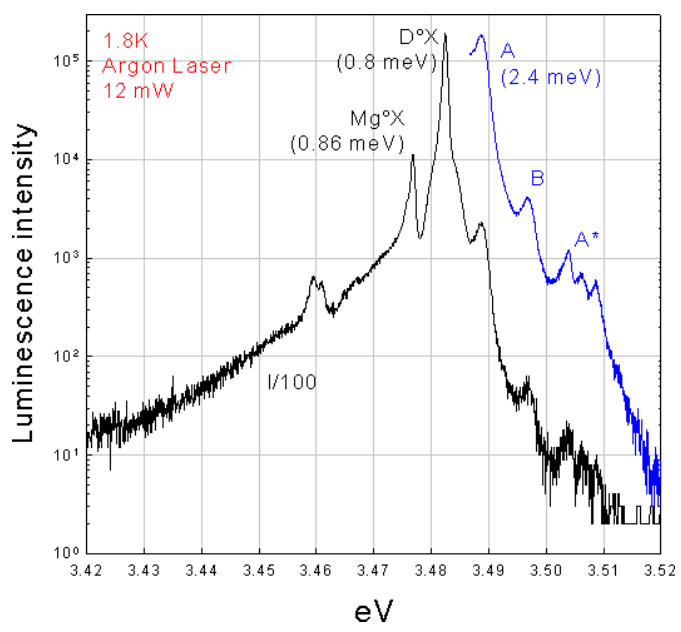

Figure 15. Low temperature luminescence spectrum measured on undoped $\mathrm{GaN}$ grown on top of a pseudo GaN substrate obtained by the to two-step overgrowth process. Bound excitons give very sharp $(\mathrm{FWHM}<1 \mathrm{meV})$ and intense peaks. Free exciton A, B and lines attributed to excited states of A are resolved. 